\title{
BMJ Open Exercise and adiposity in overweight and obese children and adolescents: protocol for a systematic review and network meta-analysis of randomised trials
}

\author{
George A Kelley, ${ }^{1}$ Kristi S Kelley, ${ }^{1}$ Russell R Pate ${ }^{2}$
}

To cite: Kelley GA, Kelley KS, Pate RR. Exercise and adiposity in overweight and obese children and adolescents: protocol for a systematic review and network meta-analysis of randomised trials. BMJ Open 2017;7:e019512. doi:10.1136/ bmjopen-2017-019512

- Prepublication history and additional material for this paper are available online. To view these files, please visit the journal online (http://dx.doi. org/10.1136/bmjopen-2017019512).

Received 7 September 2017 Revised 17 November 2017 Accepted 20 November 2017

\section{CrossMark}

${ }^{1}$ Department of Biostatistics, School of Public Health, West Virginia University, Morgantown, West Virginia, USA

${ }^{2}$ Department of Exercise Science, University of South Carolina, Columbia, South Carolina, USA

Correspondence to Professor George A Kelley; gkelley@hsc.wvu.edu

\section{ABSTRACT}

Introduction Overweight and obesity is a worldwide public health problem among children and adolescents. However, the magnitude of effect, as well as hierarchy of exercise interventions (aerobic, strength training or both), on selected measures of adiposity is not well established despite numerous trials on this issue. The primary purposes of this study are to use the network metaanalytical approach to determine the effects and hierarchy of exercise interventions on selected measures of adiposity in overweight and obese children and adolescents.

Methods and analysis Randomised exercise intervention trials $>4$ weeks, available in any language up to 31 August 2017 and which include direct and/or indirect evidence, will be included. Studies will be located by searching seven electronic databases, cross-referencing and expert review. Dual selection and abstraction of data will occur. The primary outcomes will be changes in body mass index (in $\mathrm{kg} / \mathrm{m}^{2}$ ), fat mass and percent body fat. Risk of bias will be assessed using the Cochrane Risk of Bias assessment instrument while confidence in the cumulative evidence will be assessed using the Grading of Recommendations Assessment, Development and Evaluation instrument for network meta-analysis. Network meta-analysis will be performed using multivariate random-effects metaregression models. The surface under the cumulative ranking curve will be used to provide a hierarchy of exercise treatments (aerobic, strength or both).

Ethics and dissemination This study does not require ethics approval. Findings will be presented at a professional conference and published in a peer-reviewed journal.

PROSPERO registration number CRD42017073103.

\section{INTRODUCTION}

\section{Rationale}

Overweight and obesity in children and adolescents is a major public health problem worldwide. Between 1980 and 2013, the worldwide prevalence of overweight and obesity in children and adolescents increased by $6.9 \%$, from $16.9 \%$ to $23.8 \%$, in boys and by $6.4 \%$, from $16.2 \%$ to $22.6 \%$, in girls from
Strengths and limitations of this study

- To the best of the investigative team's knowledge, this is the first systematic review to use the network meta-analytical approach to determine the effects as well as hierarchy of exercise interventions (aerobic, strength training or both) on body mass index in $\mathrm{kg} /$ $\mathrm{m}^{2}$, fat mass and percent body fat in overweight and obese children and adolescents.

- The results of this systematic review with network meta-analysis should be useful to practitioners and policy-makers for making informed decisions about exercise in the treatment of overweight and obesity in children and adolescents.

- The results of this systematic review with network meta-analysis should be useful to researchers with respect to the conduct and reporting of future research on this topic.

- Common to most meta-analyses, the results may yield significant heterogeneity which cannot be explained.

- Like any aggregate data meta-analysis, the possibility of ecological fallacy exists, that is, that group averages are not reflective of an individual's values.

developed countries. ${ }^{1}$ For developing countries, increases of $4.8 \%$, from $8.1 \%$ to $12.9 \%$ for boys and $5 \%$, from $8.4 \%$ to $13.4 \%$ in girls, were reported. ${ }^{1}$

The negative outcomes associated with obesity in children and adolescents are both immediate and long term. ${ }^{2}$ For immediacy, a population-based study of children and adolescents 5-17 years of age found that approximately $70 \%$ of obese youth had a minimum of one cardiovascular disease risk factor (high cholesterol, high blood pressure, etc). ${ }^{3}$ Obese children and adolescents are also more likely to be diagnosed with prediabetes, ${ }^{4}$ as well as being at an increased risk for bone and joint difficulties, sleep apnoea, 
and social and psychological issues such as stigmatisation, poor self-esteem and poorer health-related quality of life. ${ }^{56}$ Long-term, childhood and adolescent overweight and obesity has been demonstrated to track into adulthood, ${ }^{7-11}$ thus placing overweight and/or obese adults at a greater risk for cardiovascular disease, type 2 diabetes, stroke, several types of cancer and osteoarthritis. ${ }^{2}$

One promising intervention in the treatment of overweight and obesity is exercise. However, previous randomised trials that were limited to or included overweight and obese children and adolescents have led to conflicting results, ${ }^{12-58}$ with some reporting statistically significant reductions in adiposity (body mass index (BMI) ) as a primary outcome ${ }^{12} 131617222728314151-56$ 59-63 and others reporting no change. 1415 18-21 23-26 29 30 32-40 42-50 5758626465 When limited to overweight and obese male and female children and adolescents, ${ }^{12}$ 14 17-20 22-26 2831 $333638-4145-57$ only $18(45.0 \%)$ have reported statistically significant reductions in BMI. ${ }^{12} 172228314150-58$ While this may lead one to the general conclusion that exercise does little to reduce BMI in overweight and obese children and adolescents, this would be short-sighted since it relies on the vote-counting approach, ${ }^{66}$ an approach that has been shown to be less valid than the meta-analytical approach. ${ }^{66} 67$

Previous systematic reviews with meta-analyses that have focused on the effects of exercise as an independent intervention on BMI as a primary outcome in male and female children and adolescents have reported conflicting findings with five reporting a significant improvement in $\mathrm{BMI}^{68-72}$ and five others reporting no statistically significant improvement. ${ }^{73-77}$ However, 9 of the 10 suffer from one or more of the following limitations: (1) inclusion of a small number of studies with exercise as the only intervention, ${ }^{71} 73-75$ (2) inclusion of non-randomised trials, ${ }^{6874}$ (3) inclusion of children and adolescents who were not overweight or obese. ${ }^{70} 72747677$ Relevant to this study, all 10 suffer from reliance on pairwise versus network meta-analyses, the latter of which incorporates both direct and indirect evidence. In addition, there was an absence of an established hierarchy for determining which types of exercise (aerobic, strength training or both) might be best for improving BMI based on both direct and indirect evidence. ${ }^{68-77}$ To partially address this issue as well as demonstrate feasibility, the investigative team has recently used the network meta-analytical approach to examine the effects of exercise (aerobic, strength training or both) on BMI z-score in overweight and obese children and adolescents. ${ }^{78}$ Statistically significant reductions in BMI z-score were found for aerobic exercise and combined aerobic and strength exercise, but not strength training alone (mean, 95\% CI, aerobic, $-0.10,-0.15$ to -0.05 ; aerobic and strength, $-0.11,-0.19$ to -0.03 ; strength, $0.04,-0.07$ to 0.15$).^{79}$ Combined aerobic and strength training was ranked best, followed by aerobic exercise and then strength training. ${ }^{79}$ Consistency in evidence and risk of bias did not differ between direct and indirect studies. ${ }^{79}$ It was concluded that combined aerobic exercise and strength training as well as aerobic exercise alone are associated with reductions in BMI z-score. ${ }^{79}$ The lack of effect on BMI z-score in the strength training studies may have been the result of increases in lean muscle mass. However, since BMI in $\mathrm{kg} / \mathrm{m}^{2}$ continues to be the most frequently assessed and reported measure of adiposity in both the clinical and public health setting, such an examination using the network meta-analytical approach is needed. In addition, since all types of BMI measures as well as body weight do not capture changes in body composition (fat mass, percent body fat, etc), the inclusion of such outcomes, as previously suggested, ${ }^{79}$ is also necessary.

\section{Objectives}

The primary objectives of the current study are to conduct a systematic review with network meta-analysis of randomised trials to (1) determine the effects of exercise (aerobic, strength training or both) on adiposity (BMI in $\mathrm{kg} / \mathrm{m}^{2}$, fat mass, percent body fat) in overweight and obese children and adolescents, and (2) establish a hierarchy of exercise interventions (aerobic, strength training or both) for treating adiposity (BMI in $\mathrm{kg} / \mathrm{m}^{2}$, fat mass, percent body fat) in overweight and obese children and adolescents.

\section{METHODS \\ Overview}

This study will follow the guidelines from the Preferred Reporting Items for Systematic Reviews and Meta-Analysis extension statement for network meta-analyses of healthcare interventions. ${ }^{80}$

\section{Eligibility criteria}

The inclusion criteria for this proposed network meta-analysis will be as follows: (1) direct evidence from randomised trials that compare two or more exercise interventions (aerobic, strength training, both) or indirect evidence from randomised controlled trials that compare an exercise intervention group to a comparative control group (non-intervention, attention control, usual care, wait-list control, placebo), (2) exercise-only intervention (aerobic, strength training or both), (3) studies lasting $\geq 4$ weeks, (4) male and/or female children and adolescents $2-18$ years of age, (5) participants overweight or obese, as defined by the authors, (6) studies published in any language up to 31 August 2017, (7) data available for BMI in $\mathrm{kg} / \mathrm{m}^{2}$, fat mass or percent body fat.

Studies will be limited to randomised trials because it is the only way to control for confounders that are not known or measured as well as the observation that non-randomised controlled trials tend to overestimate the effects of healthcare interventions. ${ }^{81} 82$ Indirect evidence studies will be limited to randomised controlled trials with at least one exercise arm that participates in either aerobic, strength training, or a combination of aerobic and strength training exercise. Direct evidence 
studies will be limited to randomised trials that include at least two of the following exercise arms: (1) aerobic, (2) strength training, (3) aerobic and strength training exercise.

For the purposes of this study, exercise, aerobic exercise and strength training will be defined according to the 2008 Physical Activity Guidelines for Americans, ${ }^{83}$ that is, movement which is 'planned, structured, and repetitive and purposive in the sense that the improvement or maintenance of one or more components of physical fitness is the objective', ${ }^{83} 84$ aerobic exercise as 'exercise that primarily uses the aerobic energy-producing systems, can improve the capacity and efficiency of these systems, and is effective for improving cardiorespiratory endurance, ${ }^{83}$ and strength training as "exercise training primarily designed to increase skeletal muscle strength, power, endurance, and mass' ${ }^{83}$ Four weeks was chosen as the lower cut point for intervention length based on previous research demonstrating improvements in adiposity over this period of time in 11-year-olds. ${ }^{21}$

Participants will be limited to overweight and obese children and adolescents, as defined by the original study authors, because it has been shown that this population is at an increased risk for premature morbidity and mortality throughout their lifetime. ${ }^{85}$

While some research has suggested that studies yielding statistically significant and positive results are more likely to be published in English-language versus non-English-language journals, ${ }^{86}$ other research has shown this to not be the case. ${ }^{87}$ Given the former, studies from both English-language and non-English-language articles will be included with the latter translated into English by the second author using the freely available web-based Babelfish and Bing translators. For those studies that cannot be translated using Babelfish and/or Bing, professional translation services will be used.

BMI in $\mathrm{kg} / \mathrm{m}^{2}$ was included as one of the three primary adiposity outcomes because it is the most commonly used and understood variable by practitioners as well as others and can be easily measured from body weight and height. However, because BMI is an indirect measure of adiposity, fat mass and percent body fat will be included because they are more direct measures of adiposity. The inclusion of fat mass and percent body fat may be especially relevant for studies that include strength training given that decreases in adiposity as measured by BMI may be offset by increases in muscle mass, a secondary outcome that will be included.

\section{Information sources}

The following seven electronic databases will be searched: (1) PubMed, (2) Web of Science, (3) Cochrane Central Register of Controlled Trials, (4) Cumulative Index to Nursing and Allied Health Literature, (5) Sport Discus, (6) Translating Research into Practice and (7) ProQuest Dissertations and Theses. In addition to electronic database searches, cross-referencing will be conducted by examining the reference lists of previous review articles as well as each included study for potential articles that meet the inclusion criteria. On completion of initial searches, the third author will examine the reference list for thoroughness and completeness. Suggested studies will then be retrieved to see if they meet all inclusion criteria.

\section{Search strategy}

Search strategies specific to each database will be developed by the investigative team. Major keywords, or forms of keywords to include will be 'random', 'children', 'adolescents', 'overweight', 'obese', 'exercise,' 'physical fitness', 'body composition', 'fat mass', 'body fat', 'body composition', 'body mass index', 'adiposity'. A copy of a preliminary search strategy using PubMed, including limits, can be found in the online supplementary file. This search strategy will be adapted for other database searches. All database searches and article retrieval will be conducted by the second author with oversight from the first author.

\section{Study records}

Study selection

All studies to be screened will be imported into EndNote (V.X8; Thomson-Reuters; 2016, New York, USA) and duplicates removed electronically and then manually by the second author. A copy of the database will then be provided to the first author for duplicate screening. To minimise selection bias, the first and second authors will select all studies, independent of each other. They will then review their selections for accuracy and consistency. The full report for each article will be retrieved for all titles and abstracts that appear to meet the inclusion criteria as well as those where uncertainty exists. Multiple reports for the same study will be addressed by including the most recently published article and drawing from prior reports, assuming the same methods and sample sizes are reported. Based on previous research suggesting neither a clinically nor statistically significant effect on results, blinding to journal titles, study authors or institutions of the authors will not be employed during the screening and data abstraction processes. ${ }^{88}$ Reasons for excluded studies will be recorded using the following categories: (1) inappropriate population, (2) inappropriate intervention, (3) inappropriate comparison(s), (4) inappropriate outcome (s), (5) inappropriate study design, (6) other. On the conclusion of screening, the first and second authors will meet and review their selections. Cohen's $\kappa$ statistic will be used to measure interselection agreement. ${ }^{89}$ Any discrepancies will be resolved by consensus. If consensus cannot be reached, the third author will serve as an arbitrator. After selecting the final number of studies to include, the overall precision of the searches will be computed by dividing the number of included studies by the total number of studies screened after removing duplicates. ${ }^{90}$ The number needed-toread (NNR) will then be calculated as the reciprocal of the precision. ${ }^{90}$ A flow diagram that describes the search procedure will be included as well as supplementary file 


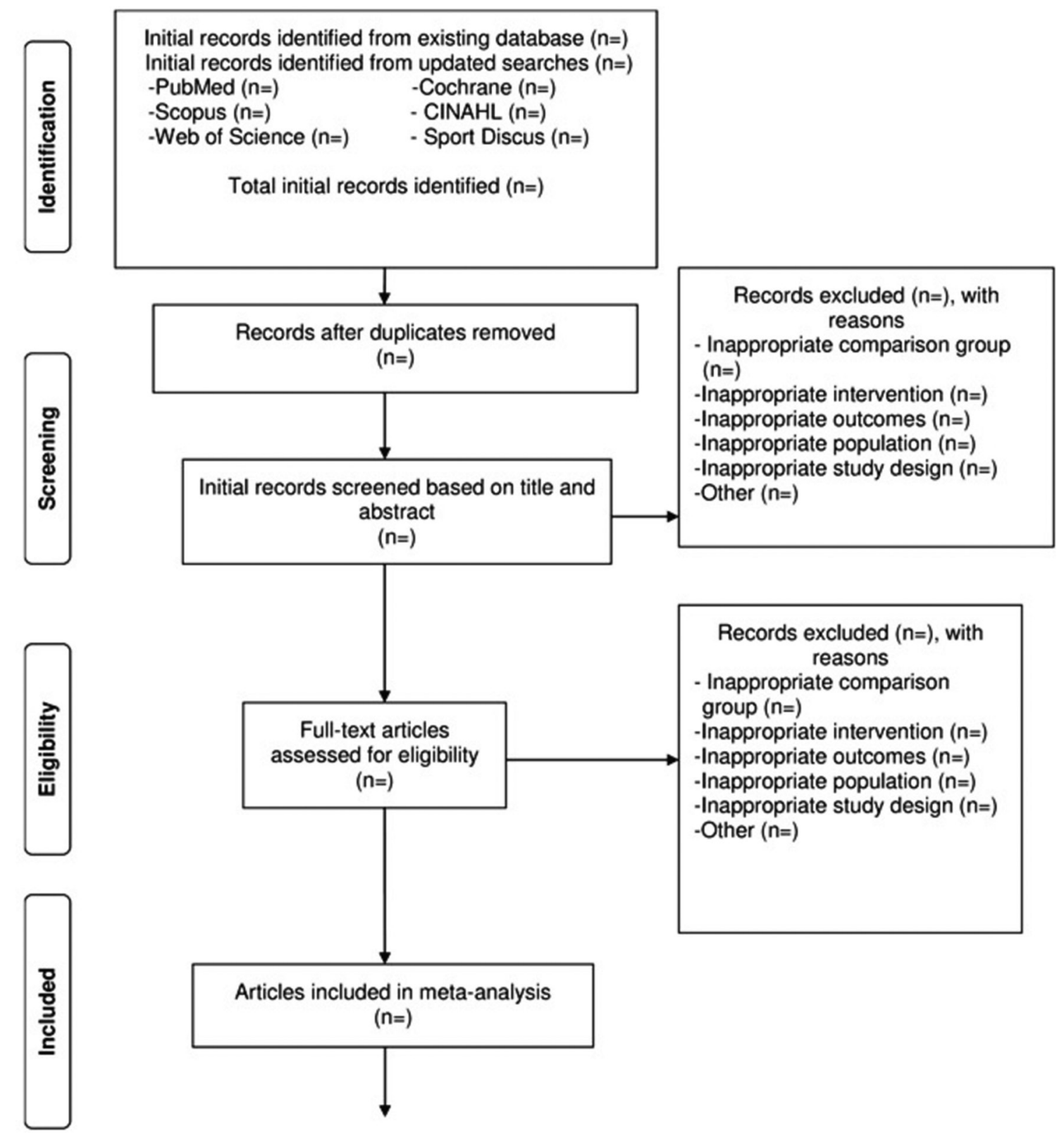

Figure 1 Proposed flow diagram to depict the search process.

a reference list of all excluded studies, including the reason(s) for exclusion. Figure 1 illustrates the proposed structure for the flow diagram.

\section{Data abstraction}

For this project, Microsoft Excel (V.2016; Microsoft Corporation, Redmond, Washington, USA) will be used to develop comprehensive electronic codebooks that will define the coding process for each of the variables coded. The codebook will be created by the first two authors with feedback from the third author. Consequently, the abstraction of data from the studies in this proposed project should require little subjective judgement on the part of the coder. The major groups of variables to code will include (1) study characteristics (author, journal, year of publication, etc), (2) participant characteristics (age, gender, height, body weight, etc) and (3) data for primary and secondary outcomes (sample sizes, baseline and postexercise means and SD, etc). Table 1 contains a preliminary list of variables that will be coded. Based on previous research by the investigative team, ${ }^{79}$ a codebook capable of including at least 242 items from each study is expected. To avoid data abstraction bias, the first two authors will independently code (dual-coding) all studies to ensure accuracy and consistency. Inter-rater agreement will be assessed using Cohen's $\kappa .{ }^{89}$ Any disagreement in the items coded will be discussed until mutual agreement is reached. If agreement cannot be reached, the third author will serve as an arbitrator.

\section{Outcomes and prioritisation}

The primary outcomes in this study will be changes in BMI in $\mathrm{kg} / \mathrm{m}^{2}$, fat mass, and percent body fat in overweight and obese children and adolescents. Secondary 
Table 1 Covariates to examine using simple meta-regression

\begin{tabular}{ll}
\hline Characteristics & Variable \\
\hline Study & $\begin{array}{l}\text { Publication year, impact factor of journal, country study conducted, type of control group, bias (sequence } \\
\text { generation, allocation concealment, blinding of participants and personnel, blinding of outcome assessors, } \\
\text { incomplete outcome data, selective outcome reporting), type of analysis }\end{array}$ \\
Participant & Age, gender, race/ethnicity, maturational stage \\
Exercise & $\begin{array}{l}\text { Type (aerobic, strength, both), length, frequency, intensity, duration, total minutes, total minutes (adjusted for } \\
\text { compliance), mode, compliance, exercise supervision, setting, number of sets, number of repetitions, rest }\end{array}$ \\
& $\begin{array}{l}\text { between sets, number of exercises, type of resistance, equipment used, fidelity (design, training, delivery, } \\
\text { receipt, enactment) }\end{array}$ \\
& $\begin{array}{l}\text { Baseline values for primary outcomes (BMl in kg/m }{ }^{2}, \text { fat mass, percent fat), method used to assess adiposity, } \\
\text { that is, instrumentation, body weight, lean body mass, waist circumference, waist-to-hip ratio, diet, energy } \\
\text { intake, energy expenditure, physical activity level, non-exercise activity, maximum oxygen consumption } \\
\text { (relative and absolute), muscular strength, resting systolic and diastolic blood pressures, total cholesterol, } \\
\text { high-density lipoprotein cholesterol, ratio of total cholesterol to high-density lipoprotein cholesterol, non-high } \\
\text { density lipoprotein cholesterol, low-density lipoprotein cholesterol, triglycerides, glycosylated haemoglobin, } \\
\text { fasting and non-fasting glucose and insulin }\end{array}$ \\
\hline
\end{tabular}

BMI, body mass index.

outcomes will include body weight, lean body mass, waist circumference, waist-to-hip ratio, energy intake, energy expenditure, physical activity level, maximum oxygen consumption (relative and absolute), muscular strength, resting systolic and diastolic blood pressures, total cholesterol, high-density lipoprotein cholesterol, ratio of total cholesterol to high-density lipoprotein cholesterol, non-high-density lipoprotein cholesterol, low-density lipoprotein cholesterol, triglycerides, glycosylated haemoglobin, fasting and non-fasting glucose and insulin.

\section{Risk of bias assessment in individual studies}

Risk of bias for included studies will be assessed using the Cochrane Risk of Bias Instrument. ${ }^{91}$ Assessment is based on judgements of low, high or unclear risk of bias across six defined domains: (1) sequence generation, (2) allocation sequence concealment, (3) blinding of participants and personnel, (4) blinding of outcome assessors, (5) incomplete outcome data and (6) selective outcome reporting. A seventh domain, whether participants were exercising regularly, as defined by the original study authors, will also be assessed using the same approach as for the other six domains. As previously recommended, study-level results will be reported for each domain according to risk of bias (low, high or unclear) while the percentage of low, high or unclear results across each domain will also be reported. ${ }^{91}$ This risk of bias approach has been recommended over the use of study quality rating scales given the lack of empirical evidence to support the latter. ${ }^{82} 9293$ Assessment for risk of bias will be limited to the primary outcomes of interest, that is, changes in BMI in $\mathrm{kg} / \mathrm{m}^{2}$, fat mass and percent body fat. All studies will be classified as high risk of bias with respect to the category 'blinding of participants and personnel' given that it's virtually impossible to blind participants to group assignment in exercise intervention protocols. Based on previous research, no study will be excluded based on risk of bias results. ${ }^{94}$

\section{Data synthesis}

\section{Calculation of effect sizes}

The primary outcomes for this study will be changes in $\mathrm{BMI}$ in $\mathrm{kg} / \mathrm{m}^{2}$, fat mass $(\mathrm{kg})$, and percent body fat using the original metric. Changes for indirect comparisons will be calculated by subtracting the change outcome difference in the exercise group minus the change outcome difference in the control group. Variances will be computed using the pooled SDs of change scores in the exercise and control groups. If change score SDs are not available, they will be calculated from 95\% CIs for either change outcome or treatment effect differences as well as pre-SD and post-SD values, the latter according to procedures developed by Follmann et al. ${ }^{95}$ For direct comparisons, that is, randomised trials with no control group, the same general procedures will be followed except that the control group data will be replaced with one of the exercise interventions as follows: (1) aerobic minus strength training, (2) aerobic and strength training combined minus aerobic training, (3) aerobic and strength training combined minus strength training. Ninety-five percent CI and $z-\alpha$ values will be calculated for each outcome from each study. For those studies that include both direct and indirect comparisons, only direct comparison data will be included since a primary purpose of the current meta-analysis is determining which exercise interventions(s) might work best for improving adiposity in children and adolescents. For studies in which adiposity outcomes are assessed at multiple intervention time points, for example, 0 weeks, 8 weeks and 16 weeks, only data from the initial and last assessment will be used. If follow-up data are available, results from such studies will also be analysed separately to determine the sustainability of changes in adiposity. If any cross-over trials are included, treatment effects will be calculated by using all assessments from the intervention and control periods and analysing them similar to a parallel group 
trial. ${ }^{96}$ While the possibility of a unit-of-analysis error exists as well as studies being underweighted versus overweighted, this method is believed to be better than alternative approaches, for example, limiting data from the first assessment point or trying to impute SDs, especially given the primary and secondary outcomes included and expected distribution of findings. ${ }^{96}$

Secondary outcomes (body weight, lean body mass, waist circumference, waist-to-hip ratio, energy intake, energy expenditure, maximum oxygen consumption (relative and absolute), resting systolic and diastolic blood pressures, total cholesterol, high-density lipoprotein cholesterol, ratio of total cholesterol to high-density lipoprotein cholesterol, non-high-density lipoprotein cholesterol, low-density lipoprotein cholesterol, triglycerides, glycosylated haemoglobin, fasting and non-fasting glucose and insulin) will be handled using the same approach as for primary outcomes. However, given the different metrics expected and the inability to convert between them, changes in physical activity levels and muscular strength will be calculated using the standardised mean difference effect size, adjusted for small sample sizes. ${ }^{97}$

\section{Pooled estimates for changes in outcomes}

Network (geometry) plots for each outcome will be used to provide a visual representation of the evidence base with nodes (circles) weighted by the number of participants randomised to each treatment and edges (lines) weighted by the number of studies evaluating each pair of treatments. ${ }^{9899}$ Contribution plots for each outcome will be used to determine the most dominant comparisons for each network estimate as well as for the entire network. ${ }^{98}$ The weights applied will be a function of the variance of the direct treatment effect and the network structure, the result being a percent contribution of each direct comparison to each network estimate. ${ }^{98}$

Network meta-analysis will be performed using multivariate random-effects meta-regression models that can be performed within a frequentist setting, allows for the inclusion of potential covariates, and correctly accounts for the correlations from multiarm trials. ${ }^{100101}$ A two-tailed $\alpha$ value $\leq 0.05$ and non-overlapping $95 \%$ CI will be considered to represent statistically significant changes. Separate network meta-analysis models will be used to examine for changes in each primary and secondary outcome. Potential covariates will be examined by (1) conducting simple meta-regression for statistically significant associations between covariates and changes in primary outcomes (BMI in $\mathrm{kg} / \mathrm{m}^{2}$, fat mass, percent fat), (2) examining for multicollinearity between covariates $(r>0.80)$ and (3) building a multiple meta-regression model. A list of potential covariates to examine using simple meta-regression is shown in table 1 . While we will include all methods used to assess adiposity, we will also conduct sensitivity analyses to see if results differ according to method of assessment, for example, fat mass assessed using whole body MRI versus bioelectrical impedance. Secondary outcomes (energy intake and expenditure, physical activity level, muscular strength) will be handled using the same approach. Transitivity, that is, similarity in the distribution of potential effect modifiers across the different pairwise comparisons for each outcome ${ }^{102}$ will include those listed in table 1. Inconsistency, that is differences in effect estimates between direct and indirect results for the same comparison, ${ }^{103}$ will be checked by assessing differences in treatment effects between direct and indirect effect estimates as well as differences between trials with different designs, for example, two-arm versus multiarm trials. ${ }^{101103104}$ However, the probability of inconsistency is considered small given recent research demonstrating that inconsistency was detected in only $2 \%-14 \%$ of tested loops, depending on the effect measure and heterogeneity estimation method. ${ }^{105} 106$ Finally, prediction intervals will be used to enhance interpretation of results with respect to the magnitude of heterogeneity as well as provide an estimate of expected results in a future study. ${ }^{107-109}$ For network meta-analysis, degrees of freedom $(d f)$ will be set to the number of studies-the number of comparisons- $1 .{ }^{109}$

\section{Meta-biases}

Small-study effects (publication bias, etc) will be assessed using comparison-adjusted funnel plots. ${ }^{98}$ In the absence of small-study effects, the comparison-adjusted funnel plot should be symmetrical around the zero line.

\section{Confidence in cumulative evidence}

Quality analysis of specific pairwise effect estimates in the network meta-analysis will be evaluated using a recently developed modification of the Grading of Recommendations Assessment, Development and Evaluation for network meta-analysis across five domains: (1) study limitations, (2) indirectness, (3) inconsistency, (4) imprecision and (5) small-study effects. ${ }^{110}$ Assessment will be conducted using the same procedures as for study selection and data abstraction.

To establish a hierarchy of exercise interventions for selected outcomes in the current meta-analysis, ranking analysis, that is, the ability to rank all interventions for a single outcome under study, for example changes in BMI in $\mathrm{kg} / \mathrm{m}^{2}$, will be used based on probabilities. However, because the ranking of treatments based exclusively on the probability of each treatment being the best should be avoided given that it does not account for the uncertainty in the relative treatment effects and the possibility for assigning higher ranks for treatments in which little evidence is available, separate rankograms and cumulative ranking probability plots will be used to present ranking probabilities along with their uncertainty for changes in primary and secondary outcomes. ${ }^{98111}$ The surface under the cumulative ranking curve (SUCRA), a transformation of the mean rank, will be used to establish a hierarchy of exercise interventions (aerobic, strength, both) while accounting for the location and variance of all treatment effects. $^{98}{ }^{111}$ Larger SUCRA values indicate better ranks for the treatment. ${ }^{98111}$ Interpretation of all rankings will 
be approached from the perspective of absolute and relative treatment effects. ${ }^{99}$

\section{Software used for statistical analysis}

All data will be analysed using Stata (V.14.1; Stata/ SE for Windows, Stata Corporation, College Station, Texas, USA), Microsoft Excel (V.2016; Microsoft Corporation, Redmond, Washington, USA), and two add-ins for Excel, SSC-Stat (V.2.18; SSC-Stat, V.3.0.; Statistical Services Center; 2007; University of Reading, UK) and EZAnalyze (V.3.0; TA Poynton; 2007).

\section{Amendments to protocol}

None to date. If this protocol is amended, the date of each amendment, a description of the change, as well as a rationale for the change, will be provided.

Contributors GAK is the guarantor. GAK, KSK and RRP drafted the manuscript. All authors contributed to the development of the data sources to search for relevant literature, including search strategy, selection criteria, data extraction criteria and risk of bias assessment strategy. GAK provided statistical expertise while RRP provided content expertise on exercise and adiposity in overweight and obese children and adolescents. All authors read, provided feedback and approved the final manuscript.

Funding This study is funded by the American Heart Association, Grant \#17GRNT33630158 (GA Kelley, Principal Investigator). The content of this manuscript is solely the responsibility of the authors and does not necessarily represent the official views of the American Heart Association.

Competing interests None declared.

Provenance and peer review Not commissioned; externally peer reviewed.

Open Access This is an Open Access article distributed in accordance with the Creative Commons Attribution Non Commercial (CC BY-NC 4.0) license, which permits others to distribute, remix, adapt, build upon this work non-commercially, and license their derivative works on different terms, provided the original work is properly cited and the use is non-commercial. See: http://creativecommons.org/ licenses/by-nc/4.0/

(C) Article author(s) (or their employer(s) unless otherwise stated in the text of the article) 2017. All rights reserved. No commercial use is permitted unless otherwise expressly granted.

\section{REFERENCES}

1. $\mathrm{Ng} \mathrm{M}$, Fleming $\mathrm{T}$, Robinson $\mathrm{M}$, et al. Global, regional, and national prevalence of overweight and obesity in children and adults during 1980-2013: a systematic analysis for the global burden of disease Study 2013. Lancet 2014;384:766-81.

2. CDC. Childhood obesity facts Atlanta. Georgia: U. S: Department of health \& human services, centers for disease control and prevention, 2015. http://www.cdc.gov/healthyschools/obesity/facts. htm (accessed 12 Mar 2015).

3. Freedman DS, Mei Z, Srinivasan SR, et al. Cardiovascular risk factors and excess adiposity among overweight children and adolescents: the bogalusa heart study. J Pediatr 2007;150:12-17.

4. Li C, Ford ES, Zhao G, et al. Prevalence of pre-diabetes and its association with clustering of cardiometabolic risk factors and hyperinsulinemia among U.S. adolescents: national health and nutrition examination survey 2005-2006. Diabetes Care 2009;32:342-7.

5. Daniels SR, Arnett DK, Eckel RH, et al. Overweight in children and adolescents: pathophysiology, consequences, prevention, and treatment. Circulation 2005;111:1999-2012.

6. Dietz WH. Overweight in childhood and adolescence. N Engl J Med 2004;350:855-7.

7. Singh AS, Mulder C, Twisk JW, et al. Tracking of childhood overweight into adulthood: a systematic review of the literature. Obes Rev 2008;9:474-88.

8. Guo SS, Chumlea WC. Tracking of body mass index in children in relation to overweight in adulthood. Am J Clin Nutr 1999;70:s145-8.
9. Freedman DS, Khan LK, Serdula MK, et al. The relation of childhood $\mathrm{BMI}$ to adult adiposity: the bogalusa heart study. Pediatrics 2005;115:22-7.

10. Freedman DS, Wang J, Thornton JC, et al. Classification of body fatness by body mass index-for-age categories among children. Arch Pediatr Adolesc Med 2009;163:805-11.

11. Freedman DS, Khan LK, Dietz WH, et al. Relationship of childhood obesity to coronary heart disease risk factors in adulthood: the Bogalusa Heart Study. Pediatrics 2001;108:712-8.

12. Alves JG, Galé CR, Souza E, et al. [Effect of physical exercise on bodyweight in overweight children: a randomized controlled trial in a Brazilian slum]. Cad Saude Publica 2008;24:S353-S59.

13. Benson AC, Torode ME, Fiatarone Singh MA. The effect of highintensity progressive resistance training on adiposity in children: a randomized controlled trial. Int J Obes 2008;32:1016-27.

14. Daley AJ, Copeland RJ, Wright NP, et al. Exercise therapy as a treatment for psychopathologic conditions in obese and morbidly obese adolescents: a randomized, controlled trial. Pediatrics 2006;118:2126-34.

15. Donnelly JE, Greene JL, Gibson CA, et al. Physical Activity Across the Curriculum (PAAC): a randomized controlled trial to promote physical activity and diminish overweight and obesity in elementary school children. Prev Med 2009:49:336-41.

16. Duncan MJ, Al-Nakeeb Y, Nevill AM. Effects of a 6-week circuit training intervention on body esteem and body mass index in British primary school children. Body Image 2009;6:216-20.

17. Farpour-Lambert NJ, Aggoun Y, Marchand LM, et al. Physical activity reduces systemic blood pressure and improves early markers of atherosclerosis in pre-pubertal obese children. J Am Coll Cardiol 2009;54:2396-406.

18. Gutin B, Owens S, Slavens G, et al. Effect of physical training on heart-period variability in obese children. $J$ Pediatr 1997:130:938-43

19. Gutin B, Owens S, Okuyama T, et al. Effect of physical training and its cessation on percent fat and bone density of children with obesity. Obes Res 1999;7:208-14.

20. Hagströmer M, Elmberg K, Mårild S, et al. Participation in organized weekly physical exercise in obese adolescents reduced daily physical activity. Acta Paediatr 2009;98:352-4.

21. Jago R, Jonker ML, Missaghian M, et al. Effect of 4 weeks of Pilates on the body composition of young girls. Prev Med 2006;42:177-80.

22. Karacabey K. The effect of exercise on leptin, insulin, cortisol and lipid profiles in obese children. J Int Med Res 2009;37:1472-8.

23. Kaufman C, Kelly AS, Kaiser DR, et al. Aerobic-exercise training improves ventilatory efficiency in overweight children. Pediatr Exerc Sci 2007;19:82-92.

24. Kelly AS, Wetzsteon RJ, Kaiser DR, et al. Inflammation, insulin, and endothelial function in overweight children and adolescents: the role of exercise. J Pediatr 2004;145:731-6.

25. Kim ES, Im JA, Kim KC, et al. Improved insulin sensitivity and adiponectin level after exercise training in obese Korean youth. Obesity 2007;15:3023-30.

26. Kim HJ, Lee S, Kim TW, et al. Effects of exercise-induced weight loss on acylated and unacylated ghrelin in overweight children. Clin Endocrinol 2008;68:416-22.

27. Kriemler S, Zahner L, Schindler C, et al. Effect of school based physical activity programme (KISS) on fitness and adiposity in primary schoolchildren: cluster randomised controlled trial. $B M J$ 2010;340:c785

28. Li YP, Hu XQ, Schouten EG, et al. Report on childhood obesity in China (8): effects and sustainability of physical activity intervention on body composition of Chinese youth. Biomed Environ $\mathrm{Sci}$ 2010;23:180-7.

29. Macias-Cervantes MH, Malacara JM, Garay-Sevilla ME, et al. Effect of recreational physical activity on insulin levels in Mexican/Hispanic children. Eur J Pediatr 2009;168:1195-202.

30. Martínez Vizcaíno V, Salcedo Aguilar F, Franquelo Gutiérrez R, et al. Assessment of an after-school physical activity program to prevent obesity among 9- to 10-year-old children: a cluster randomized trial. Int J Obes 2008;32:12-22.

31. Meyer AA, Kundt G, Lenschow $U$, et al. Improvement of early vascular changes and cardiovascular risk factors in obese children after a six-month exercise program. J Am Coll Cardiol 2006;48:1865-70.

32. Mo-suwan L, Pongprapai S, Junjana C, et al. Effects of a controlled trial of a school-based exercise program on the obesity indexes of preschool children. Am J Clin Nutr 1998;68:1006-11.

33. Murphy EC, Carson L, Neal W, et al. Effects of an exercise intervention using Dance Dance Revolution on endothelial function and other risk factors in overweight children. Int $J$ Pediatr Obes 2009;4:205-14. 
34. Neumark-Sztainer D, Story M, Hannan PJ, et al. New Moves: a school-based obesity prevention program for adolescent girls. Prev Med 2003;37:41-51.

35. Pate RR, Ward DS, Saunders RP, et al. Promotion of physical activity among high-school girls: a randomized controlled trial. $\mathrm{Am} \mathrm{J}$ Public Health 2005;95:1582-7.

36. Petty KH, Davis CL, Tkacz J, et al. Exercise effects on depressive symptoms and self-worth in overweight children: a randomized controlled trial. J Pediatr Psychol 2009;34:929-39.

37. Reilly JJ, Kelly L, Montgomery C, et al. Physical activity to prevent obesity in young children: cluster randomised controlled trial. BMJ 2006;333:1041.

38. Rooney BL, Gritt LR, Havens SJ, et al. Growing healthy families: family use of pedometers to increase physical activity and slow the rate of obesity. WMJ 2005;104:54-60.

39. Shaibi GQ, Cruz ML, Ball GD, et al. Effects of resistance training on insulin sensitivity in overweight Latino adolescent males. Med Sci Sports Exerc 2006;38:1208-15.

40. Simon C, Schweitzer B, Oujaa M, et al. Successful overweight prevention in adolescents by increasing physical activity: a 4-year randomized controlled intervention. Int J Obes 2008;32:1489-98.

41. Tan S, Yang C, Wang J. Physical training of 9- to 10-year-old children with obesity to lactate threshold intensity. Pediatr Exerc Sci 2010;22:477-85.

42. Velez A, Golem DL, Arent SM. The impact of a 12-week resistance training program on strength, body composition, and self-concept of Hispanic adolescents. J Strength Cond Res 2010;24:1065-73.

43. Walther C, Gaede L, Adams V, et al. Effect of increased exercise in school children on physical fitness and endothelial progenitor cells: a prospective randomized trial. Circulation 2009;120:2251-9.

44. Warren JM, Henry CJ, Lightowler HJ, et al. Evaluation of a pilot school programme aimed at the prevention of obesity in children. Health Promot Int 2003;18:287-96.

45. Watts K, Beye P, Siafarikas A, et al. Exercise training normalizes vascular dysfunction and improves central adiposity in obese adolescents. J Am Coll Cardiol 2004;43:1823-7.

46. Watts K, Beye P, Siafarikas A, et al. Effects of exercise training on vascular function in obese children. J Pediatr 2004;144:620-5.

47. Weintraub DL, Tirumalai EC, Haydel KF, et al. Team sports for overweight children: the Stanford Sports to Prevent Obesity Randomized Trial (SPORT). Arch Pediatr Adolesc Med 2008;162:232-7.

48. Sigal RJ, Alberga AS, Goldfield GS, et al. Effects of aerobic training, resistance training, or both on percentage body fat and cardiometabolic risk markers in obese adolescents: the healthy eating aerobic and resistance training in youth randomized clinical trial. JAMA Pediatr 2014;168:1006-14.

49. Meucci M, Cook C, Curry CD, et al. Effects of supervised exercise program on metabolic function in overweight adolescents. World $\mathrm{J}$ Pediatr 2013;9:307-11.

50. Lee S, Deldin AR, White D, et al. Aerobic exercise but not resistance exercise reduces intrahepatic lipid content and visceral fat and improves insulin sensitivity in obese adolescent girls: a randomized controlled trial. Am J Physiol Endocrinol Metab 2013;305:E1222-29.

51. Dennis BA, Ergul A, Gower BA, et al. Oxidative stress and cardiovascular risk in overweight children in an exercise intervention program. Child Obes 2013;9:15-21.

52. Maddison R, Foley L, Ni Mhurchu C, et al. Effects of active video games on body composition: a randomized controlled trial. $A m \mathrm{~J}$ Clin Nutr 2011;94:156-63.

53. Wong PC, Chia MY, Tsou IY, et al. Effects of a 12-week exercise training programme on aerobic fitness, body composition, blood lipids and C-reactive protein in adolescents with obesity. Ann Acad Med Singapore 2008;37:286-93.

54. Saygın Ouml;zcan, Saygin O, Ozturk MA. The effect of twelve week aerobic exercise programme on health related physical fitness components and blood lipids in obese girls. Afr J Pharm Pharmacol 2011;5:1441-5.

55. Sun MX, Huang XQ, Yan Y, et al. One-hour after-school exercise ameliorates central adiposity and lipids in overweight Chinese adolescents: a randomized controlled trial. Chin Med $J$ 2011;124:323-9.

56. Elloumi M, Makni E, Ounis OB, et al. Six-minute walking test and the assessment of cardiorespiratory responses during weight-loss programmes in obese children. Physiother Res Int 2011;16:32-42.

57. Lau PW, Wong delP, Ngo JK, et al. Effects of high-intensity intermittent running exercise in overweight children. Eur J Sport Sci 2015;15:182-90.

58. Alberga AS, Farnesi BC, Lafleche A, et al. The effects of resistance exercise training on body composition and strength in obese prepubertal children. Phys Sportsmed 2013;41:103-9.
59. Ackel-D'Elia C, Carnier J, Bueno CR, et al. Effects of different physical exercises on leptin concentration in obese adolescents. Int $J$ Sports Med 2014;35:164-71.

60. Fazelifar S, Ebrahim K, Sarkisian V. Effect of concurrent training and detraining on anti-inflammatory biomarker and physical fitness levels in obese children. Rev Bras Med Esporte 2013;19:349-54.

61. Ghorbanian B, Ravassi A, Kordi MR, et al. The Effects of Rope Training on Lymphocyte ABCA1 Expression, Plasma ApoA-I and HDL-c in Boy Adolescents. Int J Endocrinol Metab 2013:11:76-81.

62. Lee S, Bacha F, Hannon T, et al. Effects of aerobic versus resistance exercise without caloric restriction on abdominal fat, intrahepatic lipid, and insulin sensitivity in obese adolescent boys: a randomized, controlled trial. Diabetes 2012;61:2787-95.

63. Song JK, Stebbins CL, Kim TK, et al. Effects of 12 weeks of aerobic exercise on body composition and vascular compliance in obese boys. J Sports Med Phys Fitness 2012;52:522-9.

64. Cheng HL, Peng P, Zhu R, et al. Effects of eight weeks exercise prescription intervention on aerobic capacity, body composition, blood lipid and C-reactive protein in obese adolescents. Chinese. J Jilin Univ Med 2012;38:745-9.

65. Kelly LA, Loza A, Lin X, et al. The effect of a home-based strength training program on type 2 diabetes risk in obese Latino boys. $J$ Pediatr Endocrinol Metab 2015;28:315-22.

66. Hedges LV, Olkin I. Vote-counting methods in research synthesis. Psychol Bull 1980;88:359-69.

67. James A, Soler A, Weatherall R. Cognitive behavioural therapy for anxiety disorders in children and adolescents. Cochrane Database Syst Rev 2005;4:CD004690.

68. Schranz N, Tomkinson G, Olds T. What is the effect of resistance training on the strength, body composition and psychosocial status of overweight and obese children and adolescents? A systematic review and meta-analysis. Sports Med 2013;43:893-907.

69. Kelley GA, Kelley KS, Pate RR. Exercise and BMI in overweight and obese children and adolescents: a systematic review and trial sequential meta-analysis. Biomed Res Int 2015;2015:1-17.

70. Mei H, Xiong $\mathrm{Y}, \mathrm{Xie} \mathrm{S}$, et al. The impact of long-term school-based physical activity interventions on body mass index of primary school children - a meta-analysis of randomized controlled trials. BMC Public Health 2016;16:205.

71. García-Hermoso A, Sánchez-López M, Martínez-Vizcaíno V. Effects of aerobic plus resistance exercise on body composition related variables in pediatric obesity: a systematic review and meta-analysis of randomized controlled trials. Pediatr Exerc Sci 2015;27:431-40.

72. Dellert JC, Johnson P. Interventions with children and parents to improve physical activity and body mass index: a meta-analysis. Am J Health Promot 2014;28:259-67.

73. Atlantis E, Barnes EH, Singh MA. Efficacy of exercise for treating overweight in children and adolescents: a systematic review. Int $J$ Obes 2006;30:1027-40.

74. Harris KC, Kuramoto LK, Schulzer M, et al. Effect of school-based physical activity interventions on body mass index in children: a meta-analysis. Can Med Assoc J 2009;180:719-26.

75. McGovern L, Johnson JN, Paulo R, et al. Clinical review: treatment of pediatric obesity: a systematic review and meta-analysis of randomized trials. J Clin Endocrinol Metab 2008;93:4600-5.

76. Cesa CC, Sbruzzi G, Ribeiro RA, et al. Physical activity and cardiovascular risk factors in children: meta-analysis of randomized clinical trials. Prev Med 2014;69:54-62.

77. Guerra PH, Nobre MR, Silveira JA, et al. The effect of school-based physical activity interventions on body mass index: a meta-analysis of randomized trials. Clinics 2013;68:1263-73.

78. Kelley GA, Kelley KS. Exercise and BMI z-score in overweight and obese children and adolescents: protocol for a systematic review and network meta-analysis of randomised trials. BMJ Open 2016;6:e011258-7.

79. Kelley GA, Kelley KS, Pate RR. Exercise and BMI z-score in overweight and obese children and adolescents: a systematic review and network meta-analysis of randomized trials. J Evid Based Med 2017;10:108-28.

80. Hutton B, Salanti G, Caldwell DM, et al. The PRISMA extension statement for reporting of systematic reviews incorporating network meta-analyses of health care interventions: checklist and explanations. Ann Intern Med 2015;162:777-84.

81. Sacks H, Chalmers TC, Smith H. Randomized versus historical controls for clinical trials. Am J Med 1982;72:233-40.

82. Schulz KF, Chalmers I, Hayes RJ, et al. Empirical evidence of bias. Dimensions of methodological quality associated with estimates of treatment effects in controlled trials. JAMA 1995;273:408-12. 
83. Physical Activity Guidelines Advisory Committee. Physical activity guidelines advisory report. Washington, DC: U.S: Department of Health and Human Services, 2008.

84. Caspersen CJ, Powell KE, Christenson GM. Physical activity, exercise, and physical fitness: definitions and distinctions for health-related research. Public Health Rep 1985;100:126-31.

85. Dietz WH. Health consequences of obesity in youth: childhood predictors of adult disease. Pediatrics 1998;101:518-25.

86. Egger M, Zellweger-Zähner T, Schneider M, et al. Language bias in randomised controlled trials published in English and German. Lancet 1997;350:326-9.

87. Moher D, Pham B, Klassen TP, et al. What contributions do languages other than English make on the results of metaanalyses? J Clin Epidemiol 2000;53:964-72.

88. Berlin JA. Does blinding of readers affect the results of metaanalyses? The Lancet 1997;350:185-6.

89. Cohen J. Weighted kappa: nominal scale agreement with provision for scaled disagreement or partial credit. Psychol Bull 1968;70:213-20.

90. Lee E, Dobbins M, Decorby K, et al. An optimal search filter for retrieving systematic reviews and meta-analyses. BMC Med Res Methodol 2012;12:51.

91. Higgins JP, Altman DG, Gøtzsche PC, et al. The Cochrane Collaboration's tool for assessing risk of bias in randomised trials. BMJ 2011;343:d5928.

92. Emerson JD, Burdick E, Hoaglin DC, et al. An empirical study of the possible relation of treatment differences to quality scores in controlled randomized clinical trials. Control Clin Trials 1990;11:339-52.

93. Jüni $P$, Witschi $A$, Bloch $R$, et al. The hazards of scoring the quality of clinical trials for meta-analysis. JAMA 1999;282:1054-60.

94. Ahn S, Becker BJ. Incorporating quality scores in meta-analysis. J Educ Behav Stat 2011;36:555-85.

95. Follmann $\mathrm{D}$, Elliott $\mathrm{P}$, Suh I, et al. Variance imputation for overviews of clinical trials with continuous response. J Clin Epidemiol 1992;45:769-73.

96. Higgins JPT, Green S, eds. Cochrane handbook for systematic reviews of interventions version 5.1.0 [updated March 2011]: The Cochrane Collaboration, 2011.
97. Hedges LV, Olkin I. Statistical methods for meta-analysis. San Diego, CA: Academic Press, 1985.

98. Chaimani A, Higgins JP, Mavridis D, et al. Graphical tools for network meta-analysis in STATA. PLoS One 2013;8:e76654.

99. Catalá-López F, Tobías A, Cameron C, et al. Network meta-analysis for comparing treatment effects of multiple interventions: an introduction. Rheumatol Int 2014;34:1489-96.

100. White IR, Barrett JK, Jackson D, et al. Consistency and inconsistency in network meta-analysis: model estimation using multivariate meta-regression. Res Synth Methods 2012;3:111-25.

101. White IR. Multivariate random-effects meta-regression: updates to mvmeta. Stata J 2011;11:255-70.

102. Jansen JP, Naci H. Is network meta-analysis as valid as standard pairwise meta-analysis? It all depends on the distribution of effect modifiers. BMC Med 2013;11:159.

103. Donegan S, Williamson P, D'Alessandro U, et al. Assessing key assumptions of network meta-analysis: a review of methods. Res Synth Methods 2013;4:291-323.

104. Gb L, Ades AE. Assessing evidence inconsistency in mixed treatment comparisons. J Am Stat Assoc 2006;101:447-59.

105. Veroniki AA, Vasiliadis HS, Higgins JP, et al. Evaluation of inconsistency in networks of interventions. Int $J$ Epidemiol 2013;42:332-45.

106. Song F, Xiong T, Parekh-Bhurke S, et al. Inconsistency between direct and indirect comparisons of competing interventions: metaepidemiological study. BMJ 2011;343:d4909.

107. Higgins JP, Thompson SG, Spiegelhalter DJ. A re-evaluation of random-effects meta-analysis. J $R$ Stat Soc Ser A Stat Soc 2009;172:137-59.

108. Kelley GA, Kelley KS. Impact of progressive resistance training on lipids and lipoproteins in adults: another look at a meta-analysis using prediction intervals. Prev Med 2009;49:473-5.

109. Cooper HC, Hedges LV, Valentine JF. The handbook of research synthesis. New York: Russell Sage, 2009.

110. Salanti G, Del Giovane C, Chaimani A, et al. Evaluating the quality of evidence from a network meta-analysis. PLoS One 2014;9:e99682.

111. Salanti G, Ades AE, loannidis JP. Graphical methods and numerical summaries for presenting results from multiple-treatment metaanalysis: an overview and tutorial. J Clin Epidemiol 2011;64:163-71. 